\title{
GAUSSIAN ESTIMATES FOR DEGENERATE DIFFUSION
}

\author{
MICHAL CHOVANEC
}

(Communicated by Matthew J. Gursky)

\begin{abstract}
We prove estimates of Gaussian type for the kernel of the semigroup associated to the operator $m \triangle$, where $m$ is a positive function which may vanish at the boundary (and thus the operator may not be strongly elliptic). No regularity conditions either on the boundary of the domain or on the function $m$ are posed. The optimality of the growth condition on $m$ is discussed.
\end{abstract}

\section{INTRODUCTION}

The purpose of this article is to investigate kernel estimates for the semigroup generated by the operator $m \triangle$ on the weighted spaces $L^{p}\left(\Omega, \frac{d x}{m(x)}\right), 1 \leq p<\infty$. Here $\Omega$ is a bounded open set in $\mathbb{R}^{N}$ and $m: \Omega \rightarrow(0, \infty)$ is a bounded measurable function. Note that throughout the article we pose no regularity condition on $\partial \Omega$. We shall pose a condition on the function $m$. This is necessary in order to obtain a bounded kernel of the associated semigroup (see the remark below the proof of Theorem 3.1).

In order to get a broader view of the problems considered here we comment on the operator $m \triangle$. The operator $m \triangle$ is a particular kind of general second order elliptic operator in non-divergence form having merely a principal part

$$
\sum_{i, j=1}^{N} a_{i j}(x) \frac{\partial u}{\partial x_{i} \partial x_{j}} .
$$

Here, the coefficients $a_{i j}$ take a special form $a_{i i}(x)=m(x)$ for $i=1, \ldots, N$ and $a_{i j}(x) \equiv 0$ for $i \neq j$. The operator is isotropic since the coefficients do not depend on a particular direction.

Note that the operator $m \triangle$ is elliptic (in the terminology of [10]) since we assume that $m(x)>0$, but we do not assume strict ellipticity (this would require $m(x)>\varepsilon$ for some $\varepsilon>0)$.

The main result of the present paper is the following.

Theorem 1.1. Let $\Omega$ be an open bounded subset of $\mathbb{R}^{N}$ and $m: \Omega \rightarrow(0, \infty)$ be a bounded measurable function such that $\frac{1}{m} \in L^{q}(\Omega, d x)$ for some $q>\max \{N / 2,1\}$. Then $m \triangle$ generates a strongly continuous semigroup on $L^{p}\left(\Omega, \frac{d x}{m(x)}\right)$ for $1 \leq p<\infty$. There exists a bounded measurable function $K(t, x, y)$ (called a kernel) such that

Received by the editors October 25, 2010 and, in revised form, May 11, 2011.

2010 Mathematics Subject Classification. Primary 35K65, 35K08, 47D06.

Key words and phrases. Heat equation, degenerate diffusion, kernel estimates, semigroups. 
for any $u \in L^{2}\left(\Omega, \frac{d x}{m(x)}\right)$ we have the representation

$$
\left(e^{m \triangle t} u\right)(x)=\int_{\Omega} K(t, x, y) u(y) \frac{d x}{m(y)}, \quad \text { for a.a. } x \in \Omega .
$$

Furthermore, the kernel satisfies the following bound:

$$
0 \leq K(t, x, y) \leq C_{\hat{N}, q} \cdot t^{-\frac{\hat{N}(q-1)}{2 q-\hat{N}}} e^{-\frac{|x-y|^{2}}{4 \hat{c} t}} \text { for all } t>0,
$$

where the constant $\tilde{c}$ depends only on $\|m\|_{\infty}$. The constant $\hat{N}$ is equal to the dimension $N$ in case $N \geq 3$; otherwise $(N=1,2)$ the constant $\hat{N}$ may be chosen arbitrarily subject to $2<\hat{N}<2 q$.

The investigation of Gaussian estimates in the classical setting of the Laplace operator has been a subject of many papers and is well-documented in the literature. We refer the reader to [3], 6], 8], [12] and the references therein. On the other hand, there seems to be comparatively little known about degenerate operators of the form considered here, since the operator at hand is not strongly elliptic and has only measurable coefficients. However, for a particular form of the perturbing function, namely for a regularised version of the distance function and its powers, basic time estimates for the kernel were obtained in [13. There, however, the underlying domain is assumed to be smooth. We stress that we pose no condition on the boundary of $\Omega$.

We should also mention that estimates for operators of the form $m A$, where $A$ is a strictly elliptic second order differential operator, have been considered in 9 . There, however, the condition $m>\varepsilon>0$ is posed. Thus for a particular case of a Laplace operator, our result supersedes those in 9 .

We comment that a theory of the regularity of boundary points for the operator $m \triangle$ on the space of continuous functions vanishing at the boundary of $\Omega$ was developed in [2].

We describe the content of the sections. We start in Section 2 by reviewing the definition of the operator $m \triangle$ and stating a generation result on weighted $L^{p}$-spaces. We proceed in Section 3 by proving a basic ultracontractive estimate. Section 4 is concerned with ultracontractivity for the twisted operator, building on the usual Davies method. Finally, in Section 5, Gaussian estimates for $m \triangle$ are proved. We should like to comment that the methods used to prove our main result are to a large extent known; however, they seem not to have been exploited in this depth previously.

\section{Forms on A Weighted SPACE}

In this section we introduce a general sesquilinear form acting on the weighted space $L^{2}\left(\Omega, \frac{d x}{m(x)}\right)$, where $\Omega$ is an open and bounded set in $\mathbb{R}^{N}$ and $m: \Omega \rightarrow(0, \infty)$ is a bounded measurable function such that $\frac{1}{m} \in L^{q}(\Omega, d x)$ for some $q>\max \{N / 2,1\}$. The space $L^{2}\left(\Omega, \frac{d x}{m(x)}\right)$ is a Hilbert space where the scalar product is given by

$$
\langle u \mid v\rangle=\int_{\Omega} u(x) \overline{v(x)} \frac{d x}{m(x)} .
$$

We need the following lemma.

Lemma 2.1. Under the assumptions stated above the space $H_{0}^{1}(\Omega)$ is dense and continuously embedded in $L^{2}\left(\Omega, \frac{d x}{m(x)}\right)$. 
Proof. We have

$$
\begin{aligned}
\int_{\Omega} \frac{|u|^{2}}{m(x)} d x & \leq\left(\int_{\Omega} \frac{1}{m(x)^{q}} d x\right)^{\frac{1}{q}}\left(\int_{\Omega}|u|^{2 q^{\prime}} d x\right)^{\frac{1}{q^{\prime}}} \\
& \leq c_{N}\left\|\frac{1}{m}\right\|_{L^{q}(\Omega)}\left(\int_{\Omega}|\nabla u|^{2}\right)^{\frac{1}{2}} \leq c_{N}\left\|\frac{1}{m}\right\|_{L^{q}(\Omega)}\|u\|_{H_{0}^{1}(\Omega)},
\end{aligned}
$$

where we used the Hölder inequality and a Sobolev embedding (i.e. Theorem 7.10 or 7.15 in [10] depending on the dimension $N$ ).

On $L^{2}\left(\Omega, \frac{d x}{m(x)}\right)$ we define the operator $m \triangle_{2}$ by

$$
\begin{aligned}
\mathcal{D}\left(m \triangle_{2}\right) & :=\left\{u \in H_{0}^{1}(\Omega): \exists f \in L^{2}\left(\Omega, \frac{d x}{m(x)}\right) \text { such that } \triangle u=\frac{f}{m}\right\}, \\
\left(m \triangle_{2}\right) u & :=f .
\end{aligned}
$$

Note that $\frac{f}{m} \in L^{1}(\Omega)$ since we have

$$
\int_{\Omega} \frac{|f(x)|}{m(x)} d x \leq\left(\int_{\Omega}|f(x)|^{2} \frac{d x}{m(x)}\right)^{\frac{1}{2}}\left(\int_{\Omega} \frac{d x}{m(x)}\right)^{\frac{1}{2}} .
$$

Thus the identity $\triangle u=\frac{f}{m}$ is well-defined in $\mathcal{D}(\Omega)^{\prime}$. The expression $m \triangle_{2}$ is purely symbolic and has to be understood in the sense of the above definition. In fact, in general $\triangle u$ is merely in $\mathcal{D}(\Omega)^{\prime}$ and $m \triangle u$ cannot be defined as a distribution.

We will understand $m \triangle_{2}$ as the operator associated to a sesquilinear form.

Let $\mathbf{a}: \Omega \rightarrow \mathbb{C}^{N \times N}, b: \Omega \rightarrow \mathbb{C}^{N}, c: \Omega \rightarrow \mathbb{C}^{N}$ and $a_{0}: \Omega \rightarrow \mathbb{C}$ be bounded measurable functions. We also assume the following ellipticity condition on the matrix a: there exists a constant $c_{E}$ such that

$$
\operatorname{Re} \sum_{k, l=1}^{N} a_{k l}(x) \xi_{k} \overline{\xi_{l}} \geq c_{E}|\xi|^{2}
$$

for all $\xi \in \mathbb{C}^{N}$ and almost all $x \in \Omega$.

Then we define a sesquilinear form $a$ by

$$
\begin{aligned}
a(u, v): & =\int_{\Omega} \nabla u(x) \cdot[\mathbf{a}(x) \overline{\nabla v(x)}] d x+\int_{\Omega} b(x) \cdot \nabla u(x) \overline{v(x)} d x \\
& +\int_{\Omega} u(x) c(x) \cdot \overline{\nabla v(x)} d x+\int a_{0}(x) u(x) \overline{v(x)} d x
\end{aligned}
$$

with the domain $\mathcal{D}(a)=H_{0}^{1}(\Omega)$. Here the dot product of two $N$-dimensional vectors $w$ and $z$ is given by $w \cdot z:=\sum_{j=1}^{N} w_{j} z_{j}$.

Since throughout the work we assume the function $m$ to be bounded from above, we have

Lemma 2.2. $L^{2}\left(\Omega, \frac{d x}{m(x)}\right)$ is continuously embedded in $L^{2}(\Omega, d x)$.

Proof. This follows immediately from the assumption of boundedness (from above) of $m$.

In order to prove continuity of the form $a$ we shall make use of the following (sectoriality) estimate. 
Lemma 2.3. There exists a constant $w$ which is of the form

$$
w:=c_{a, N, m}\left(\||b|+|c|\|_{\infty}^{2}+\left\|a_{0}\right\|_{\infty}\right)
$$

such that

$$
|\operatorname{Im} a(u, u)| \leq c_{\boldsymbol{a}} \operatorname{Re} a(u, u)+w\|u\|_{L^{2}\left(\Omega, \frac{d x}{m(x)}\right)}^{2} .
$$

Here $c_{\boldsymbol{a}}$ is a constant depending on the matrix $\boldsymbol{a}$. It may be chosen as $c_{\boldsymbol{a}}:=3$ in case $\boldsymbol{a}$ equals the identity matrix.

Proof. We estimate

$$
\begin{aligned}
\operatorname{Re} a(u, u) & =\operatorname{Re} \int_{\Omega} \nabla u(x) \cdot \mathbf{a}(x) \overline{\nabla u(x)} d x+\int_{\Omega} \operatorname{Re}(b(x)+c(x)) \operatorname{Re}(\bar{u}(x) \nabla u(x)) d x \\
& -\int_{\Omega} \operatorname{Im}(b(x)-c(x)) \operatorname{Im}(\bar{u}(x) \nabla u(x)) d x+\int_{\Omega} a_{0}(x)|u(x)|^{2} d x \\
& \geq c_{E} \int_{\Omega}|\nabla u(x)|^{2} d x-\left\|\operatorname{Re} a_{0}\right\|_{\infty} \int_{\Omega}|u(x)|^{2} d x \\
& -\sqrt{N}\left(\|\operatorname{Re}(b+c)\|_{\infty}+\|\operatorname{Im}(c-b)\|_{\infty}\right) \int_{\Omega}|\bar{u}(x) \nabla u(x)| d x \\
& \geq c_{E} \int_{\Omega}|\nabla u(x)|^{2} d x-\frac{1}{2} c_{E} \int_{\Omega}|\nabla u(x)|^{2} d x \\
& -\left(\frac{N}{c_{E}}\left(\|\operatorname{Re}(b+c)\|_{\infty}^{2}+\|\operatorname{Im}(c-b)\|_{\infty}^{2}\right)+\left\|\operatorname{Re} a_{0}\right\|_{\infty}\right) \int_{\Omega}|u(x)|^{2} d x,
\end{aligned}
$$

where we used the inequality $|2 v w| \leq \frac{1}{c_{E}} v^{2}+c_{E} w^{2}$ for $v, w \in \mathbb{R}$. We may also write $\operatorname{Im} a(u, u)=\operatorname{Im} \int_{\Omega} \nabla u(x) \cdot \mathbf{a}(x) \overline{\nabla u(x)} d x+\int_{\Omega} \operatorname{Re}(b(x)-c(x)) \operatorname{Im}(\bar{u}(x) \nabla u(x)) d x$

$$
+\int_{\Omega} \operatorname{Im}(b(x)+c(x)) \operatorname{Re}(\bar{u}(x) \nabla u(x)) d x+\int_{\Omega} \operatorname{Im} a_{0}(x)|u(x)|^{2} d x
$$

and estimate

$$
\begin{aligned}
|\operatorname{Im} a(u, u)| & \leq\|a\|_{\infty} \int_{\Omega}|\nabla u(x)|^{2} d x+\left\|\operatorname{Im} a_{0}\right\|_{\infty} \int_{\Omega}|u(x)|^{2} d x \\
& +\sqrt{N}\left(\|\operatorname{Re}(b-c)\|_{\infty}+\|\operatorname{Im}(b+c)\|_{\infty}\right) \int_{\Omega}|\bar{u}(x) \nabla u(x)| d x \\
& \leq\left(\|a\|_{\infty}+\frac{1}{2}\right) \int_{\Omega}|\nabla u(x)|^{2} d x \\
& +\left(N\|\operatorname{Re}(b-c)\|_{\infty}^{2}+\|\operatorname{Im}(b+c)\|_{\infty}^{2}+\left\|\operatorname{Im} a_{0}\right\|_{\infty}\right) \int_{\Omega}|u(x)|^{2} d x .
\end{aligned}
$$

Combining the estimates for $\operatorname{Re} a(u, u)$ and $\operatorname{Im} a(u, u)$ we obtain

$$
|\operatorname{Im} a(u, u)| \leq c_{\mathbf{a}} \operatorname{Re} a(u, u)+c_{\mathbf{a}, N}\left(\||b|+|c|\|_{\infty}^{2}+\left\|a_{0}\right\|_{\infty}\right) \int_{\Omega}|u(x)|^{2} d x .
$$

The last estimate and Lemma 2.2 conclude the proof.

Remark 2.4. We point out here the fact that the proof of Lemma 2.3 follows the usual proof, valid in the non-weighted setting. What is important here is only the 
validity of the embedding $L^{2}\left(\Omega, \frac{d x}{m(x)}\right) \hookrightarrow L^{2}(\Omega, d x)$ guaranteed by the assumption of boundedness from above the function $m$.

Remark 2.5. Since it will be useful later, we note that in the course of the proof of Lemma 2.3 we also obtained the estimate

$$
\int_{\Omega}|\nabla u(x)|^{2} d x \leq c_{\mathbf{a}} \operatorname{Re} a(u, u)+w\|u\|_{L^{2}\left(\Omega, \frac{d x}{m(x)}\right)}^{2} .
$$

We see from the results above that if we define in $L^{2}\left(\Omega, \frac{d x}{m(x)}\right)$ a form

$$
a_{w}(u, v):=a(u, v)+w\langle u, v\rangle_{L^{2}\left(\Omega, \frac{d x}{m(x)}\right)} \text { with } \mathcal{D}(a):=H_{0}^{1}(\Omega),
$$

then $a_{w}$ is a densely defined, accretive, closed and continuous sesquilinear form in $L^{2}\left(\Omega, \frac{d x}{m(x)}\right)$ (the continuity is seen by applying the well-known Schwarz's inequality; see Proposition 6.1 in the Appendix). Therefore, by the theory of sesquilinear forms (see e.g. Chapter I in [12), there exists an associated operatol $A_{w}$ which is the generator of a contractive $C_{0}$-semigroup on $L^{2}\left(\Omega, \frac{d x}{m(x)}\right)$.

For the sake of reference we summarise what we have done in the following theorem.

Theorem 2.6. Let $\Omega$ be a bounded open subset of $\mathbb{R}^{N}$ and $m: \Omega \rightarrow(0, \infty)$ be a bounded measurable function such that $\left.\frac{1}{m} \in L_{(}^{q} \Omega, d x\right)$ for some $q>\max \{N / 2,1\}$. Let

$$
a: \Omega \rightarrow \mathbb{C}^{N \times N}, b: \Omega \rightarrow \mathbb{C}^{N}, c: \Omega \rightarrow \mathbb{C}^{N} \text { and } a_{0}: \Omega \rightarrow \mathbb{C}
$$

be bounded measurable functions such that (2) holds. Define $a(\cdot, \cdot)$ by (3). Then there exists $w \in \mathbb{R}_{+}$such that $a_{w}(\cdot, \cdot)$ defined by (6) is a densely defined, accretive, closed and continuous sesquilinear form in $L^{2}\left(\Omega, \frac{d x}{m(x)}\right)$. The associated operator $A_{w}$ is the generator of a contractive $C_{0}$-semigroup on $L^{2}\left(\Omega, \frac{d x}{m(x)}\right)$. The constant $w$ may be chosen to be of the form

$$
w:=c_{\boldsymbol{a}, N}\|m\|_{\infty}\left(\||b|+|c|\|_{\infty}^{2}+\left\|a_{0}\right\|_{\infty}\right) .
$$

We shall now see that in the case $\mathbf{a} \equiv I$ and $b \equiv c \equiv a_{0} \equiv 0$ (in which case we may take $w=0$ ) the operator $A_{0}$ equals $-m \triangle_{2}$ (with the Dirichlet boundary condition).

In fact, for $u, f \in L^{2}\left(\Omega, \frac{d x}{m(x)}\right)$ we have by definition,

$$
\begin{array}{cc}
u \in \mathcal{D}(A) \text { and }-A u=f & \text { if and only if } \\
a(u, v)=-\int_{\Omega} f(x) v(x) \frac{d x}{m(x)} & \text { for all } v \in V .
\end{array}
$$

Taking $v \in \mathcal{D}(\Omega)$, this implies that $\triangle u=\frac{f}{m}$. Hence $u \in \mathcal{D}\left(m \triangle_{2}\right)$ and $\left(m \triangle_{2}\right) u=f$. Conversely, if $u \in \mathcal{D}\left(m \triangle_{2}\right)$ and $\left(m \triangle_{2}\right) u=f$, then $\triangle u=\frac{f}{m}$ in $\mathcal{D}(\Omega)^{\prime}$. Since $u \in H_{0}^{1}(\Omega)$, this implies that

$$
\int_{\Omega} \nabla u(x) \nabla v(x) d x=-\langle\triangle u, v\rangle=-\int_{\Omega} f(x) v(x) \frac{d x}{m(x)}
$$

for all $v \in \mathcal{D}(\Omega)$. Since $\mathcal{D}(\Omega)$ is dense in $V$ it follows that $u \in \mathcal{D}(A)$ and $A u=f$.

We shall use the general theory of this section (i.e. non-zero $b, c$ and $a_{0}$ ) to prove Gaussian estimates via the twisted form.

\footnotetext{
${ }^{1}$ We shall denote by $A:=A_{0}$ the operator associated to the (unshifted) form $a(\cdot, \cdot)$.
} 


\section{THE TWISTED FORM}

We start this section with the following ultracontractivity estimate. For clarity of exposition we formulate the result separately for dimensions $N=1$ and $N=2$.

Theorem 3.1. a) Let $\Omega$ be a bounded open set in $\mathbb{R}^{N}, N \geq 3$, and $m: \Omega \rightarrow(0, \infty)$ such that $\frac{1}{m} \in L^{q}(\Omega)$ for some $q>\frac{N}{2}$. Then the semigroup $e^{m \triangle t}$ is ultracontractive. Moreover, there exists $c>0$ s.t.

$$
\left\|e^{m \triangle t} f\right\|_{\infty} \leq c \cdot t^{-\frac{N(q-1)}{2(2 q-N)}}\|f\|_{L^{2}\left(\Omega, \frac{d x}{m(x)}\right)}
$$

for all $f \in L^{2}\left(\Omega, \frac{d x}{m(x)}\right)$ and all $t>0$.

b) Let $\Omega$ be a bounded open set in $\mathbb{R}^{N}, N=1$ or $N=2$, and let $m: \Omega \rightarrow(0, \infty)$ be such that $\frac{1}{m} \in L^{q}(\Omega)$ for some $q>1$. Then the semigroup $e^{m \triangle t}$ is ultracontractive. Moreover, for each $\alpha>\frac{1}{2}$ there exists a constant $c_{\alpha}$ such that

$$
\left\|e^{m \triangle t} f\right\|_{\infty} \leq c_{\alpha} \cdot t^{-\alpha}\|f\|_{L^{2}\left(\Omega, \frac{d x}{m(x)}\right)}
$$

for all $f \in L^{2}\left(\Omega, \frac{d x}{m(x)}\right)$ and all $t>0$.

For the proof of Theorem 3.1 we need the definition of a submarkovian semigroup and a characterisation of ultracontractivity for such semigroups.

Consider a Borel measure $\eta$ on $\Omega$. A (bounded linear) operator $S$ on $L^{2}(\Omega, d \eta)$ is called submarkovian if $f(x) \leq 1$ a.e. implies $S f(x) \leq 1$ a.e. This is equivalent to saying that $S$ is positive and

$$
\|S f\|_{\infty} \leq\|f\|_{\infty} \text { for all } f \in L^{2}(\Omega, d \eta) \cap L^{\infty}(\Omega, d \eta) .
$$

To say that a semigroup $e^{A t}$ is submarkovian means that each $e^{A t}, t \geq 0$, is submarkovian.

We have the following characterisation of ultracontractivity.

Theorem 3.2. Let $e^{A t}$ be a submarkovian semigroup on $L^{2}(\Omega, d \eta)$. If $\mu>2$, then a bound of the form

$$
\left\|e^{A t} u\right\|_{\infty} \leq c t^{-\frac{\mu}{4}}\|u\|_{L^{2}(\Omega, d \eta)}
$$

for all $t>0$ and all $u \in L^{2}(\Omega, d \eta)$ is equivalent to a bound of the form

$$
\|u\|_{L^{\frac{2 \mu}{\mu-2}}(\Omega, d \eta)}^{2} \leq c_{2} a(u, u)
$$

for all $u \in \mathcal{D}(a)$.

Proof. See e.g. 6], Theorem 2.4.2.

Proof of Theorem 3.1. First, we comment that the semigroup $e^{m \triangle t}$ is submarkovian. This is easily checked using the well-known Beurling-Deny criteria. See [2] for details (cf. also the footnote at the end of this section).

Define $\hat{N}:=N$ if $N \geq 3$; otherwise let $\hat{N}>2$ be arbitrary subject to the condition $\hat{N}<2 q$. We check (10). Take $u \in \mathcal{D}(a)=H_{0}^{1}(\Omega)$, set $\mu:=\frac{2 \hat{N}(q-1)}{2 q-\hat{N}}$ and 
estimate

$$
\begin{aligned}
\|u\|_{L^{\frac{2 \mu}{\mu-2}}\left(\Omega, \frac{d x}{m(x)}\right)}^{2} & =\left[\int_{\Omega}|u(x)|^{\frac{2 \mu}{\mu-2}} \frac{d x}{m(x)}\right]^{\frac{\mu-2}{\mu}} \\
& \leq\left[\left(\int_{\Omega} \frac{d x}{m(x)^{q}}\right)^{\frac{1}{q}}\left(\int_{\Omega}|u(x)|^{\frac{2 \mu q^{\prime}}{\mu-2}} d x\right)^{\frac{1}{q^{\prime}}}\right]^{\frac{\mu-2}{\mu}} \\
& =c(m, \hat{N}, q) \cdot\left(\int_{\Omega}|u(x)|^{\frac{2 \hat{N}}{\hat{N}-2}} d x\right)^{\frac{1}{q^{\prime}} \frac{\mu-2}{\mu} \frac{\hat{N}-2}{2 \hat{N}} \frac{2 \hat{N}}{\hat{N}-2}} \\
& =c(m, \hat{N}, q)\left(\int_{\Omega}|u(x)|^{\frac{2 \hat{N}}{\hat{N}-2}} d x\right)^{\frac{(\hat{N}-2)}{2 \hat{N}}} \\
& \leq \tilde{c}(m, \hat{N}, q) \int_{\Omega}|\nabla u(x)|^{2} d x=c \cdot a(u, u),
\end{aligned}
$$

where Hölder's inequality, the equality $\frac{\mu-2}{\mu} \frac{q-1}{q} \frac{\hat{N}}{\hat{N}-2}=\frac{2 q(\hat{N}-2)}{2 \hat{N}(q-1)} \frac{q-1}{q} \frac{\hat{N}}{\hat{N}-2}=1$, and a Sobolev inequality (i.e. Theorem 7.10 or 7.15 in [10] depending on the dimension $N$ ) were used. We conclude the proof by applying Theorem 3.2 .

At this point we should comment that in dimensions larger than two the condition $q>\frac{N}{2}$ is in this generality (arbitrary bounded domains) optimal (up to $\geq$ possibly). That is, for any $q<\frac{N}{2}$ there exist a domain $\Omega$ and a function $m$ with $\frac{1}{m} \in L^{q}(\Omega)$ such that the kernel of the semigroup $e^{m \triangle t}$ is not bounded. See [5] or [4] for details. Thus we shall assume $\frac{1}{m} \in L^{q}(\Omega)$ for some $q>\frac{\hat{N}}{2}$ in the rest of the paper.

Our goal here is to obtain a Gaussian estimate for $e^{m \Delta t}$. In order to do so, we use the Davies method. Fix $x_{0} \in \Omega, a \in \mathbb{R}^{d}$ with $\|a\|=1$ and define

$$
\phi(x)=\phi_{x_{0}, a}(x):=\left\langle x-x_{0}, a\right\rangle,
$$

where $\langle\cdot, \cdot\rangle$ is the usual scalar product in $\mathbb{R}^{d}$.

Introduce the form

$$
a_{\lambda}(u, v):=a\left(e^{\lambda \phi} u, e^{-\lambda \phi} v\right) \quad \text { for } \quad u, v \in H_{0}^{1}(\Omega) .
$$

The form $a_{\lambda}$ satisfies the assumptions of Section 2 with

$$
\begin{aligned}
b(x): & =-\lambda \nabla \phi(x), \\
c(x): & =\lambda \nabla \phi(x), \\
a_{0}(x): & =-\lambda^{2}\langle\nabla \phi(x), \nabla \phi(x)\rangle .
\end{aligned}
$$

We also note that the adjoint form to $a_{\lambda}$ looks the same as $a_{\lambda}$ only the roles of $b$ and $c$ interchange.

The constant $w$ in Lemma 2.3 is of the form $w_{\lambda}=\tilde{c} \cdot \lambda^{2}$, where $\tilde{c}$ depends only on $N$ and $\|m\|_{\infty}$. Therefore, if we denote by $A_{\lambda}$ the operator associated with $a_{\lambda}$, we have

$$
\left\|e^{A_{\lambda} t-w_{\lambda} t}\right\|_{\mathcal{L}\left(L^{2}\left(\Omega, \frac{d x}{m(x)}\right)\right)} \leq 1 .
$$

Here $\mathcal{L}(X)$ denotes the space of bounded linear operators on a Banach space $X$ equipped with the usual norm.

Now we investigate under which assumption we may obtain a contractive semigroup on $L^{\infty}\left(\Omega, \frac{d x}{m(x)}\right)$. There are well-known criteria for $L^{\infty}$-contractivity, which 
may be expressed in terms of the associated form (see e.g. paragraph 4.3 in [12]). In particular, from Corollary 4.8 in 12 , we se 2 that upon choosing

$$
w_{\infty, \lambda}:=\lambda^{2}|\nabla \phi(x)|^{2}+\lambda \triangle \phi(x)=\lambda^{2}\|a\|^{2}+0=\lambda^{2}
$$

we have

$$
\left\|e^{A_{\lambda} t-w_{\lambda, \infty} t}\right\|_{\mathcal{L}\left(L^{\infty}\left(\Omega, \frac{d x}{m(x)}\right)\right)} \leq 1 .
$$

By interpolation we get

$$
\left\|e^{A_{\lambda} t-\max \left(w_{\lambda}, w_{\lambda, \infty}\right) t}\right\|_{\mathcal{L}\left(L^{p}\left(\Omega, \frac{d x}{m(x)}\right)\right.} \leq 1 \text { for any } p \in[2, \infty] .
$$

Since an analogous estimate also holds for the adjoint form $a_{\lambda}^{*}$, we finally obtain by duality

$$
\left\|e^{A_{\lambda} t-w_{\lambda} t}\right\|_{\mathcal{L}\left(L^{p}\left(\Omega, \frac{d x}{m(x)}\right)\right.} \leq 1
$$

for all $1 \leq p \leq \infty$ with $w_{\lambda}:=\tilde{c} \lambda^{2}$, where $\tilde{c}$ is a constant larger than (or equal to) one and otherwise depending only on $\|m\|_{\infty}$.

\section{UltraCONTRACTIVITY FOR THE TWISTED FORM}

We start with the estimate

$$
\|u\|_{L^{\frac{2 \mu}{\mu-2}}\left(\Omega, \frac{d x}{m(x)}\right)}^{2} \leq c_{1} \int_{\Omega}|\nabla u(x)|^{2} d x,
$$

where $\mu:=\frac{2 \hat{N}(q-1)}{2 q-\hat{N}}$ and $\hat{N}:=N$ in case $N \geq 3$ and $\hat{N}$ is arbitrary subject to the condition $2<\hat{N}<2 q$ in cases $N=1$ or $N=2$. The estimate (12) was obtained in the proof of Theorem 3.1 .

We now prove a hypercontractive estimate for the twisted semigroup.

Theorem 4.1. For the semigroup $A_{\lambda}$ and for $w_{\lambda}$ defined in (111) we have

$$
\left\|e^{A_{\lambda} t-w_{\lambda} t}\right\|_{L^{2} \rightarrow L^{\eta}} \leq c_{2} t^{-\frac{1}{2}} \text { for all } t>0,
$$

where $\eta:=\frac{2 \mu}{\mu-2}$ and $L^{2} \rightarrow L^{\eta}$ denotes the norm of a linear operator from $L^{2}\left(\Omega, \frac{d x}{m(x)}\right)$ to $L^{\eta}\left(\Omega, \frac{d x}{m(x)}\right)$.

Proof. Combining (12) and (5) we have

$$
\|u\|_{L^{\eta}\left(\Omega, \frac{d x}{m(x)}\right)}^{2} \leq c_{2}\left(\operatorname{Re} a_{\lambda}(u, u)+w_{\lambda}\langle u, u\rangle_{m}\right),
$$

where $\langle\cdot, \cdot\rangle_{m}$ denotes the scalar product on $L^{2}\left(\Omega, \frac{d x}{m(x)}\right)$.

\footnotetext{
${ }^{2}$ Strictly speaking, the setting in 12 is non-weighted; however, the result remains true for the weighted $L^{p}$-spaces because the underlying form is the same. This has been explicitly computed and confirmed in 4]; it is stated as Corollary 2.2.4 there.
} 
Define $S(t):=e^{A_{\lambda} t-w_{\lambda} t}$. Then $\|S(t)\|_{L^{\eta} \rightarrow L^{\eta}} \leq 1$, and for $f \in L^{2}\left(\Omega, \frac{d x}{m(x)}\right) \cap$ $L^{\eta}\left(\Omega, \frac{d x}{m(x)}\right)$ we have

$$
\begin{gathered}
c_{2}^{-1} t\|S(t) f\|_{L^{\eta}\left(\Omega, \frac{1}{m(x)}\right)}^{2}=c_{2}^{-1} \int_{0}^{t}\|S(t) f\|_{L^{\eta}}^{2} d s=c_{2}^{-1} \int_{0}^{t}\|S(s) S(t-s) f\|_{L^{\eta}}^{2} d s \\
\leq c_{2}^{-1} \int_{0}^{t}\|S(s) f\|_{L^{\eta}}^{2} d s \leq \int_{0}^{t} \operatorname{Re} a_{\lambda}(S(s) f, S(s) f)+w_{\lambda}\langle S(s) f, S(s) f\rangle_{m} d s \\
=\int_{0}^{t}-\frac{1}{2} \frac{d}{d s}\|S(s) f\|_{L^{2}\left(\Omega, \frac{d x}{m(x)}\right)}^{2} d s=\frac{1}{2}\left(\|f\|_{2}^{2}-\|S(t) f\|_{2}^{2}\right) \leq \frac{1}{2}\|f\|_{L^{2}\left(\Omega, \frac{d x}{m(x)}\right)}^{2} .
\end{gathered}
$$

Since such functions $f$ are dense in $L^{2}\left(\Omega, \frac{d x}{m(x)}\right)$, the conclusion of the theorem is proved.

Now by a well-known procedure we may prove ultracontractivity for the twisted semigroup.

Theorem 4.2. There exists a constant $c_{3}$ independent of $\lambda$ such that

$$
\left\|e^{A_{\lambda} t-w_{\lambda} t}\right\|_{L^{2} \rightarrow L^{\infty}} \leq c_{3} \cdot t^{-\frac{\mu}{4}} \text { for all } t>0 .
$$

Proof. For the proof we refer the reader to Lemma 6.1 in [12].

Since an analogous theorem may be proved for $A_{\lambda}^{*}$ (the corresponding form is of the same type; only the coefficients $b$ and $c$ are interchanged), we obtain by duality:

Theorem 4.3. There exists a constant $c_{4}$ independent of $\lambda$ such that

$$
\left\|e^{A_{\lambda} t-w_{\lambda} t}\right\|_{L^{1} \rightarrow L^{\infty}} \leq c_{4} \cdot t^{-\frac{\mu}{2}} \text { for all } t>0 .
$$

This is equivalent to the following estimate for the kernel $K_{\lambda}(t, x, y)$ of the semigroup $e^{A_{\lambda} t}$ :

$$
\left|K_{\lambda}(t, x, y)\right| \leq c_{4} \cdot t^{-\frac{\mu}{2}} \cdot e^{\tilde{c} \lambda^{2}} \text { for all } t>0 .
$$

The constant $\tilde{c}$ depends only on $\|m\|_{\infty}$.

Proof. The first part of the theorem has been proved above. The second part follows from the well-known Dunford-Pettis theorem (see e.g. [1]).

\section{Gaussian estimates fOR $m \triangle$}

In order to prove Gaussian estimates we need to estimate the kernel of the operator $e^{-\lambda \phi} e^{m \triangle} e^{\lambda \phi}$, where $\phi=\phi_{x_{0}, a}$ is the smooth function defined in the middle of Section 3. However, the associated form to this operator is the form $a_{\lambda}$ investigated in the previous two sections. Therefore we have by Theorem 4.3

$$
\left\|e^{-\lambda \phi} e^{m \triangle} e^{\lambda \phi}\right\|_{L^{1}\left(\Omega, \frac{d x}{m(x)}\right) \rightarrow L^{\infty}\left(\Omega, \frac{d x}{m(x)}\right)} \leq c_{4} \cdot t^{-\frac{\mu}{2}} \cdot e^{\tilde{c} \lambda^{2} t} \text { for all } t>0 .
$$

This implies an estimate for the kernel $K(t, x, y)$ of $e^{m \triangle}$ :

$$
0 \leq K(t, x, y) \leq C \cdot t^{-\frac{\mu}{2}} \cdot e^{\tilde{c} \lambda^{2} t+\lambda(\phi(x)-\phi(y))}
$$

for all $t>0$. 
Setting $\lambda:=\frac{\phi(y)-\phi(x)}{2 \tilde{c} t}$ and then optimizing over $a$ in the definition of $\phi$ we finally obtain the bound

$$
0 \leq K(t, x, y) \leq C \cdot t^{-\frac{\mu}{2}} e^{-\frac{|x-y|^{2}}{4 \tilde{c} t}} \text { for all } t>0 .
$$

We summarize in the main theorem.

Theorem 5.1. Let $\Omega$ be an open bounded subset of $\mathbb{R}^{N}$ and $m: \Omega \rightarrow(0, \infty)$ be a bounded measurable function such that $\frac{1}{m} \in L^{q}(\Omega, d x)$ for some $q>\max \{N / 2,1\}$. Then we have the following estimate for the kernel $K(t, x, y)$ of the operator $e^{m \triangle t}$ :

$$
0 \leq K(t, x, y) \leq C_{\hat{N}, q} \cdot t^{-\frac{\hat{N}(q-1)}{2 q-\hat{N}}} e^{-\frac{|x-y|^{2}}{4 \bar{c} t}} \quad \text { for all } t>0,
$$

where the constant $\tilde{c}$ depends only on $\|m\|_{\infty}$. The constant $\hat{N}$ is equal to the dimension $N$ in case $N \geq 3$; otherwise $(N=1,2)$ the constant $\hat{N}$ may be chosen arbitrarily subject to $2<\hat{N}<2 q$.

\section{Appendix}

Proposition 6.1. Let a be a sesquilinear form on a Hilbert space $H$. If there exists a constant $c$ such that

$$
|\operatorname{Im} a(u, u)| \leq c \operatorname{Re} a(u, u), \quad \forall u \in \mathcal{D}(a),
$$

then the inequality

$$
|a(u, v)| \leq(c+1) \sqrt{\operatorname{Re} a(u, u)} \sqrt{\operatorname{Re} a(v, v)}
$$

is valid for all $u, v \in \mathcal{D}(a)$.

Proof. See p. 310 in 11 .

\section{ACKNOWLEDGEMENTS}

We would like to acknowledge financial support from the Graduiertenkolleg 1100: Modellierung, Analyse und Simulation in der Wirtschaftsmathematik at the University of Ulm. The author also thanks the anonymous referee for valuable advice.

\section{REFERENCES}

[1] W. Arendt, A.V. Bukhvalov, Integral representations of resolvents and semigroups, Forum Math. 6 (1994), 111-135. MR1253180 (94k:47062)

[2] W. Arendt, M. Chovanec, Dirichlet regularity and degenerate diffusion, Trans. Amer. Math. Soc. 362.11 (2010), 5861-5878. MR2661499 (2011f:35143)

[3] W. Arendt, A.F.M. ter Elst, Gaussian estimates for second order elliptic operators with boundary conditions, J. Operator Theory 38.1 (1997), 87-130. MR.1462017 (98k:47094)

[4] M. Chovanec, Degenerate Diffusion - Behaviour at the Boundary and Kernel Estimates, $\mathrm{PhD}$ thesis, University of Ulm, 2010.

[5] M. Chovanec, Ultracontractivity for the degenerate diffusion, Ulmer Seminare, 2007/08.

[6] E. B. Davies, Heat Kernels and Spectral Theory, University Press, Cambridge, 1989. MR.990239 (90e:35123)

[7] E. B. Davies, $L^{1}$ properties of second order elliptic operators, Bull. London Math. Soc. 17 (1985), 417-436. MR806008 (87g:58126)

[8] E. B. Davies, B. Simon, Ultracontractivity and the heat kernel for Schrödinger operators and Dirichlet Laplacians, J. Funct. Anal. 59 (1984), 335-395. MR.766493 (86e:47054)

[9] X. T. Duong, E. M. Ouhabaz, Complex multiplicative perturbations of elliptic operators: Heat kernel bounds and holomorphic functional calculus, Diff. Integ. Eq. 12 (1999), no. 3, 395-418. MR1674426 (2000a:47096) 
[10] D. Gilbarg, N. S. Trudinger, Elliptic Partial Differential Equations of Second Order, Springer, 1983. MR737190 (86c:35035)

[11] T. Kato, Perturbation Theory for Linear Operators, Springer, Berlin-Heidelberg-New York, 1980.

[12] E. M. Ouhabaz, Analysis of heat equations on domains, Princeton University Press, Princeton, NJ, 2005. MR2124040 (2005m:35001)

[13] M. M. H. Pang, $L^{\perp}$ properties of two classes of singular second order elliptic operators, J. London Math. Soc. 38 (1988), 525-543. MR.972136 (90b:35168)

Graduiertenkolleg 1100, University of Ulm, 89081 Ulm, Germany

E-mail address: michal.chovanec@uni-ulm.de

Current address: Departement Mathematik, HG J 43, ETH Zürich, Rämisstrasse 101, 8092 Zürich, Switzerland 